\title{
Bullying escolar y aprendizaje en los estudiantes \\ del cuarto y quinto grado de secundaria, 2021
}

\author{
Álvarez Blas De Aroni Sara \\ saritabogchimb@hotmail.com \\ ORCID: $\underline{\text { 0000-0003-0689-3711 }}$ \\ Groberti Alfredo Medina Corcuera \\ grome@ucvvirtual.edu.pe \\ https://orcid.org/0000-0003-4035-157X \\ Universidad César Vallejo \\ Chimbote - Perú
}

\section{RESUMEN}

La presente investigación tuvo como objetivo general determinar la relación entre el bullying escolar y el aprendizaje de los estudiantes del cuarto y quinto grado de educación secundaria de la institución educativa Micaela Bastidas en el año 2021. El tipo de investigación fue basica, con un diseño de investigación no experimental - correlacional, de alcance temporal transversal; la muestra estuvo conformada por 85 estudiantes de las secciones "A" y "B" del cuarto y quinto grado de secundaria matriculados en el año 2021, a quienes se les aplicó un cuestionario para evaluar la variable bullying y una ficha de captación de datos para recabar información de la variable aprendizaje, se llegó a concluir: Se determinó relación inversa y significativa $(\mathrm{Rho}=-0.696$; sig. $=0.000)$ entre el bullying escolar y el aprendizaje de los estudiantes del cuarto y quinto grado de secundaria de la I.E. Micaela Bastidas - Santa Ancash año 2021, dando a conocer que a menor bullying escolar se podrá tener un mejor aprendizaje de los estudiantes, de la misma se procedió con el rechazo de la hipótesis nula.

Palabras claves: bullying escolar; comportamiento agresivo; conductas inadecuadas; aprendizaje. 


\title{
School bullying and learning in fourth and fifth grade students - institución educativa Micaela Bastidas, 2021
}

\begin{abstract}
The present investigation had as general objective to determine the relationship between school bullying and the learning of students of the fourth and fifth grade of secondary education of the educational institution Micaela Bastidas in the year 2021. The type of investigation was basic, with a design of non-experimental research - correlational, of transversal temporal scope; The sample consisted of 85 students from sections "A" and "B" of the fourth and fifth grade of secondary school enrolled in 2021, to whom a questionnaire was applied to evaluate the bullying variable and a data collection form to To collect information on the learning variable, it was concluded: An inverse and significant relationship was determined $($ Rho $=-0.696$; sig. $=0.000)$ between school bullying and the learning of students in the fourth and fifth grade of EI. Micaela Bastidas - Santa Ancash year 2021, making it known that the less school bullying it will be possible to have a better learning of the students, the same proceeded with the rejection of the null hypothesis.
\end{abstract}

Keywords: school bullying; aggressive behavior; inappropriate behavior; learning.

Artículo recibido: 02 noviembre. 2021 Aceptado para publicación: 28 noviembre 2021 Correspondencia: saritabogchimb@ hotmail.com Conflictos de Interés: Ninguna que declarar 


\section{INTRODUCCIÓN}

El bullying o acoso escolar siempre ha tenido presencia en la escuela, sobre todo en la tradicional, en el que la violencia, además de los estudiantes, lo ejercía también el docente. Con los cambios sociales y educativos la escuela ha impregnado su accionar de enfoques y principios que buscan la inclusión y la atención a la diversidad con el fin de romper el círculo repetitivo de la violencia escolar. Según Briones-Zambrano (2020) los estudiantes que han sido víctimas de acoso u otros tipos de intimidación tienden a reproducir ese comportamiento como un mecanismo para revertir dicha situación. Para el referido autor los estudiantes con "características físicas, condiciones financieras o socioculturales diferentes de la mayoría terminan siendo discriminados", otro factor sería el bajo rendimiento académico que presentan algunos estudiantes, que, frente a una posible amenaza que representan los estudiantes con calificaciones más altas, muchas veces se traducen en actitudes ofensivas.

Este interés por erradicar la violencia del ámbito escolar ha conllevado a múltiples investigaciones en varios países, para conocer cómo se manifiesta este problema social, qué factores están asociados o bien realizar o experimentar propuestas. Así se tiene el estudio de Ferrel et ál. (2015) que detectó entre los estudiantes de Santa Martha Colombia, dos factores de riesgo asociados a la violencia, la impulsividad y la soledad, pero, además identificó a las habilidades sociales como un factor protector.

El Departamento de Salud y Servicios Humanos de los Estados Unidos (2021) informa que, en este país, aproximadamente el $22 \%$ de los estudiantes entre 12 a 18 años fueron víctimas de acoso y que entre los grados 9-12 manifiestan que han sido acosados en los ambientes escolares en el año anterior al estudio. Por su parte, el Centro Nacional de Educación detectó que en el año 2016 más del 20\% de los estudiantes reconocieron haber sido víctimas de acoso, este porcentaje es preocupante teniendo en cuenta que estos actos de violencia afectan, de variadas formas, el bienestar de los estudiantes (Patterson, 2020). El estudio realizado en Noruega por Olweus (1993), citado en Fretwell (2015) identificó que, en las escuelas primarias y secundarias de dicho país, el 15 por ciento de los 84.000 estudiantes que participaron del estudio fueron víctimas de situaciones de intimidación, este porcentaje representa una proporción de uno de cada siete estudiantes. Fretwell (2015) manifiesta que las consecuencias de estos actos de acoso e intimidación son 
inmediatas y pueden tener repercusiones hasta la edad adulta, tales como la depresión, uso de sustancias o dificultades en sus relaciones sociales y afectivas.

Para Alonso (2018) "el acoso escolar para el autor está presente en todos los niveles educativos", empieza con hostigamientos y luego derivan en actos agresivos como las burlas o agresiones físicas que suelen darse de manera prolongada. Para Rojas (2019) estos actos en la escuela devienen de la violencia que se da en la sociedad peruana, expresada en las agresiones verbales y físicas que cotidianamente se observan en las calles, tanto entre adultos como jóvenes. Por tanto, combatir el bullying o violencia escolar pasa por mejorar el entorno social.

Es preocupante que la violencia afecte a los estudiantes, yendo en contra de su derecho a desarrollarse en ambientes libres de violencia y maltrato, cuando la violencia se instaura en las escuelas se destruye el equilibrio del sistema educativo afectando las relaciones interpersonales. Esta preocupación generó que el Ministerio de Educación implemente la plataforma SiseVe, para la denuncia de situaciones de bullying escolar en los espacios educativos, una herramienta para romper con el círculo de abuso y maltrato que muchas veces era soslayado en las comunidades educativas, asimismo se están implementando documentos, estrategias y materiales para prevenir y atender la violencia escolar. (Minedu, 2017b)

Según el Ministerio de Educación, en el año 2019, se reportaron, a través de la plataforma virtual del SiseVe, 27 niños por día que fueron víctimas de actos de violencias en sus escuelas, en la primera mitad del año en mención se realizaron 4,931 denuncias de maltrato en los colegios, cifra alta en comparación a los 2,384 registrados en el mismo periodo del año 2018, al revisar el número registrado en los últimos 7 años se observa una preocupante tendencia al alza.

Entre los estudiantes de la I.E. "Micaela Bastidas" el problema del bullying es latente, causando preocupación los actos violentos entre pares, utilizando palabras ofensivas, insultos, apodos o sobrenombres, acción de maltrato físico o maltrato psicológico o verbal, alejándolos del aula o equipo de trabajo bajo amenazas, imponiendo una brecha con los demás; por lo que hay necesidad de establecer el nivel de relación entre este problema del bullying escolar y el aprendizaje de los estudiantes para explicar las consecuencias de este fenómeno que deja una huella indeleble en los estudiantes que han sido víctimas u observadores en el ambiente escolar, los resultados permitirán avizorar la 
necesidad de evitar acciones de imposición corporal, verbal, psicológica, de un estudiante hacia el par elegido para maltratarlo con frecuentes agresiones.

Por lo expuesto, el problema que busca absolver esta investigación es qué relación hay entre el bullying y el aprendizaje en los estudiantes del cuarto y quinto grado de secundaria de la Institución Educativa Micaela Bastidas en el año 2021 en la Provincia del Santa-Ancash, esta interrogante se plantea debido a que los casos de bullying se manifiestan en estos grados de estudio que coincide con una edad en que los estudiantes son muy vulnerables en la configuración de su autoestima personal.

La investigación se justifica en la necesidad de conocer las implicancias del bullying en el aprendizaje de los estudiantes ya que, al conocer dicha relación, se podrá proyectar acciones de contingencia frente a estas conductas y brindar el soporte emocional para evitar los bajos niveles de rendimiento o deserción escolar.

Asimismo, como toda investigación que aplica procedimientos e instrumentos para recabar información, el presente estudio proveerá de instrumentos y metodologías para los docentes o directivos que tengan interés en diagnosticar situaciones de bullying en sus aulas; el instrumento utilizado, es una propuesta que puede ser adaptada según el contexto y grupo poblacional al que se dirige, previa opinión de expertos.

El estudio contribuyó al campo del conocimiento al aportar resultados sobre el comportamiento de la variable en un escenario concreto, en el que se ubica la institución educativa que alberga a los estudiantes que conformaron la muestra. Realidades similares existen en la provincia del Santa, para los cuales existen las posibilidades de emplear los procedimientos e instrumentos validados.

Es importante, señalar que el objetivo general de esta investigación fue Determinar la relación entre el bullying escolar y el aprendizaje de los estudiantes del cuarto y quinto grado de secundaria de la I.E. Micaela Bastidas - Santa Ancash año 2021; los objetivos específicos han sido: Estudiar la relación entre el comportamiento agresivo y el aprendizaje de los estudiantes del cuarto y quinto grado de secundaria de la I.E. Micaela Bastidas - Santa Ancash año 2021; Conocer la relación entre el comportamiento antisocial y el aprendizaje de los estudiantes del cuarto y quinto grado de secundaria de la I.E. Micaela Bastidas - Santa Ancash año 2021; Establecer la relación entre la desinterés e indisciplina en el aula y el aprendizaje de los estudiantes del cuarto y quinto grado de secundaria de la I.E. Micaela Bastidas - Santa Ancash año 2021; Estudiar la 
relación entre las conductas inadecuadas y el aprendizaje de los estudiantes del cuarto y quinto grado de secundaria de la I.E. Micaela Bastidas - Santa Ancash año 2021.

Las hipótesis de investigación fueron: Existe relación inversa y significativa entre bullying escolar y aprendizaje de los estudiantes, además se estableció una hipótesis nula no existe relación inversa entre bullying escolar y aprendizaje de los estudiantes.

El estudio realizado por Monelos (2015), sobre la violencia escolar en estudiantes de nivel secundario, tuvo como objetivo conocer la presencia de bullying y ciberbullying en estudiantes en Coruña España, empleó el diseño de tipo descriptivo- no experimental transversal, con una muestra de 538 estudiantes adolescentes entre hombres y mujeres. Utilizó una encuesta sobre bullying. Se llegaron a las siguientes conclusiones: no existe un nivel alto de violencia estudiantil, dado que entre el $88 \%$ y $97.10 \%$ de estudiantes manifiesta no tener comportamiento de agresividad por bullying ni de ciberbullying; el $32.69 \%$ guarda silencio al ser víctima de agresión o bullying., si lo expresa, un $61 \%$ de ellos lo comunica a los docentes mientras y un $72,5 \%$ a su familia; no existe un espacio escolar específico en donde ocurren los hechos de violencia escolar; los estudiantes varones, a diferencia de las mujeres, son agredidos con actos violentos, físicas y verbales; el 37.4\% ha sido víctima de agresión verbal en cuanto a apodos; el 15.9\% discriminó a los de menor edad; el $13 \%$ por insultos.

Se cuenta con los aportes de Amaya (2017) quien investigó sobre las alternativas no violentas que generan las comunidades educativas en Colombia, ante el acoso escolar que afecta a los estudiantes en su diversidad. El Objetivo fue determinar competencias sobre el respeto a la comunidad educativa, valorando a sus miembros sin discriminación, con la inclusión y erradicación de la violencia entre pares. La investigación fue aplicada, con un diseño no experimental, con una población de 3.600 de estudiantes entre 11 y 14 años y una muestra de 185 estudiantes, 50 docentes y 87 padres de familia. Se utilizaron entrevistas, red de discusión y talleres para recabar los datos antes y después de terminar el estudio. Se concluyó que, la intimidación en la escuela se manifiesta en poner adjetivos o apodos y el robo intimidatorio; el acoso entre profesor-alumno, lo que genera malestar en el ambiente escolar; los estudiantes al presenciar el acoso escolar usaron el pensamiento crítico como estrategia para erradicar el maltrato escolar interviniendo con acciones no violentas o agresivas. 


\section{ESTRATEGIAS METODOLÓGICAS O MATERIALES Y MÉTODOS}

La investigación fue de tipo básica porque se han estudiado las variables en su estado natural sin generar cambios o introducir variables experimentales. Para Baena (2017), esta investigación, obedeciendo al criterio según su objetivo, corresponde a un tipo de investigación que se basa en fuentes teóricas existentes. Por otro lado, se considera de enfoque cuantitativo, porque plantea objetivos y se responde con resultados estadísticos que permiten su análisis.

El diseño de investigación es no experimental porque no se manipula a las variables, considerándose correlacional porque con la información de las variables estudiadas, a través de un procedimiento estadístico, se estableció el nivel de correlación entre ambas variables. Este diseño de investigación también ha permitido relacionar las dimensiones de las variables. A decir de Hernández, Fernández y Baptista (2016) los estudios correlacionales permiten comparar un concepto o una variable al conocer el comportamiento de otras variables vinculadas, en el presente estudio la relación entre dos variables.

El alcance temporal es transversal, porque la información necesaria para el estudio se recolectó en un solo momento.

Según Baena (2017) la población se conformó por las personas cuyas características se centran en lo común dentro de un grupo social, situándose en un mismo espacio y podrían diferenciarse con el tiempo. Asimismo, puede determinarse como un conjunto de estructuras: individuales y materiales; factibles de una relación en común que comparten el ambiente o espacio establecido en el tiempo, la población estuvo constituida por estudiantes de matriculados en la I.E "Micaela Bastidas” durante el año escolar 2021.

\section{Tabla 1}

Población del estudio

\begin{tabular}{lccc}
\hline \multirow{2}{*}{ Estudiantes } & Varones & Mujeres & Total \\
\cline { 2 - 4 } & 200 & 156 & \\
\hline
\end{tabular}

Fuente: SIAGIE: 2021

\section{Muestra}

Para Sánchez, Reyes, y Mejía, (2018) la muestra es el "conjunto de casos o individuos extraídos de una población por algún sistema de muestreo probabilístico o no probabilístico" (p. 93). Por tanto, las muestras son sub conjuntos de elementos o casos 
extraídos del conjunto de la población a través de métodos o procedimientos racionales y de interés del investigador.

\section{Tabla 2}

Muestra

\begin{tabular}{lccc}
\multicolumn{1}{c}{ Estudiantes } & Varones & Mujeres & Total \\
\hline $4^{\circ}$ grado A & 13 & 9 & 22 \\
$4^{\circ}$ grado B & 10 & 10 & 22 \\
$5^{\circ}$ grado A & 10 & 8 & 18 \\
$5^{\circ}$ grado B & 8 & 10 & 18 \\
\hline
\end{tabular}

\section{Total}

Fuente: SIAGIE 2021

La muestra la conformaron 80 estudiantes del cuarto y quinto grado de secundaria sección “A” y "B" matriculados en el año 2021 y quienes tuvieron las posibilidades de responder el cuestionario Google proporcionando la información requerida para la presente investigación.

\section{Muestreo}

Se ha empleado un tipo de muestreo no probabilístico intencional, llamado también por García (2017) muestreo discrecional; en este caso los sujetos que van ser parte del estudio son elegidos deliberadamente, según el criterio del investigador, quien considera que determinados sujetos, son los más adecuados para la investigación.

En este caso, los estudiantes incluidos en la investigación han sido los de cuarto y quinto año de secundaria debido a que han desarrollado cursos con la investigadora y por tanto puede tener ascendencia sobre ellos para la aplicación del instrumento, además, son estudiantes que ya han pasado por la experiencia de la formación presencial del primer al tercer año de secundaria. No se eligieron los grados inferiores debido a las mínimas y algunos casos $\left(1^{\circ}\right.$ y $\left.2^{\circ}\right)$ nula experiencia de educación presencial.

\section{RESULTADOS Y DISCUSIÓN}

En esta parte del estudio se detalla los niveles alcanzados por las dimensiones y las variables seleccionadas para el estudio para dar a conocer la percepción que se tiene, mostrando lo siguiente: 


\section{Tabla 3}

Frecuencia del bullying escolar

\begin{tabular}{|c|c|c|c|c|c|c|c|c|}
\hline \multirow{2}{*}{$\begin{array}{c}\text { Dimensiones y } \\
\text { variable }\end{array}$} & \multicolumn{2}{|c|}{ Bajo } & \multicolumn{2}{|c|}{ Regular } & \multicolumn{2}{|c|}{ Alto } & \multicolumn{2}{|c|}{ Total } \\
\hline & fi & $\%$ & fi & $\%$ & fi & $\%$ & fi & $\%$ \\
\hline $\begin{array}{l}\text { Comportamiento } \\
\text { agresivo }\end{array}$ & 23 & $28.8 \%$ & 45 & $56.3 \%$ & 12 & $15.0 \%$ & 80 & $100.0 \%$ \\
\hline $\begin{array}{l}\text { Comportamiento } \\
\text { antisocial }\end{array}$ & 23 & $28.8 \%$ & 51 & $63.8 \%$ & 6 & $7.5 \%$ & 80 & $100.0 \%$ \\
\hline $\begin{array}{l}\text { Desinterés e } \\
\text { indisciplina en el aula }\end{array}$ & 32 & $40.0 \%$ & 44 & $55.0 \%$ & 4 & $5.0 \%$ & 80 & $100.0 \%$ \\
\hline Conductas inadecuadas & 22 & $27.5 \%$ & 52 & $65.0 \%$ & 0 & $7.5 \%$ & 80 & $100.0 \%$ \\
\hline Bullying escolar & 24 & $30.0 \%$ & 50 & $62.5 \%$ & 6 & $7.5 \%$ & 80 & $100.0 \%$ \\
\hline
\end{tabular}

Fuente: Instrumento aplicado a los estudiantes

\section{Figura 1}

\section{Barra del bullying escolar}

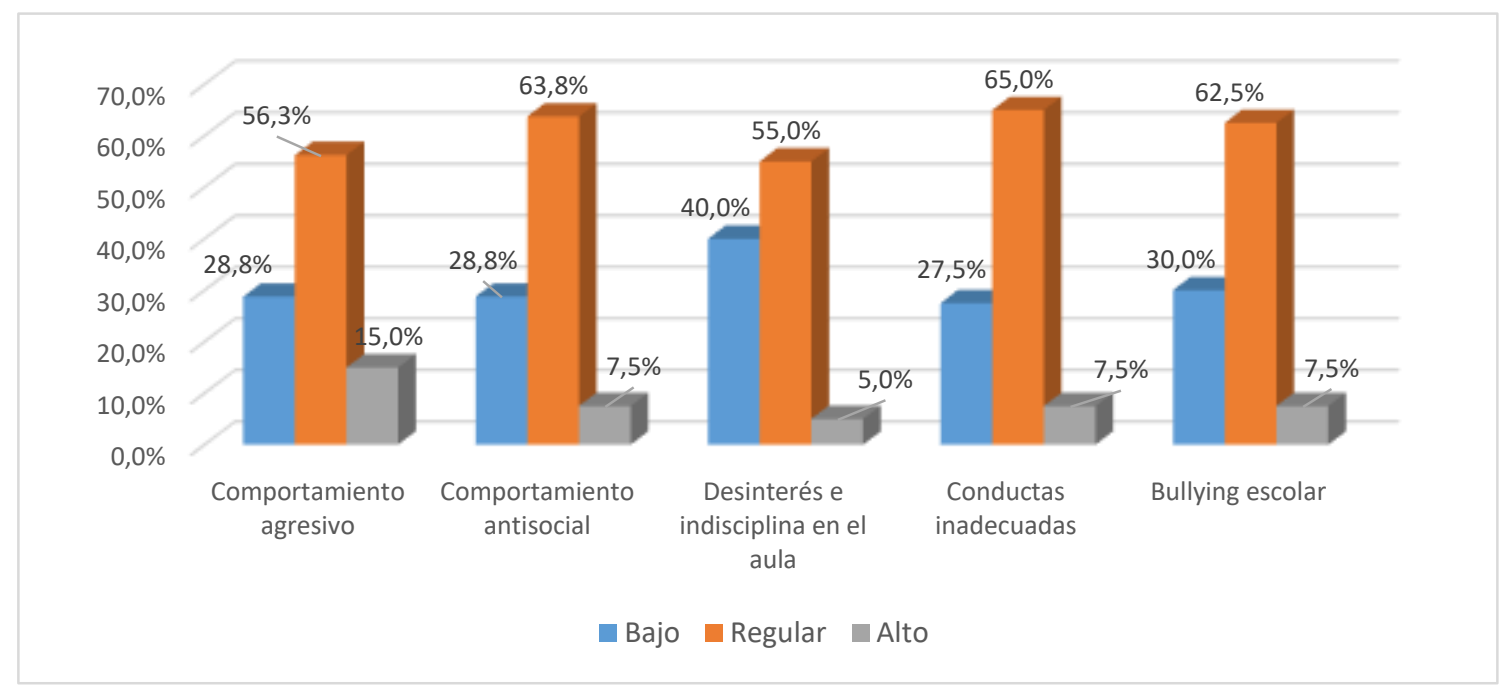

\section{Interpretación}

Por medio del análisis realizado de la aplicación del instrumento a los estudiantes se tiene para el comportamiento agresivo que 23 estudiantes que corresponden al $28.8 \%$ opinan que es bajo, luego se tiene a 45 estudiantes que conforman el $56.3 \%$ lo evalúan como regular, finalmente se tiene a 12 estudiantes que integran el $15.0 \%$ opinan que es alto. Al realizar el análisis del comportamiento antisocial se podo ubicar que 23 estudiantes que representan el $28.8 \%$ lo encuentran como bajo, de ahí se tiene que 51 estudiantes que representan el $63.8 \%$ opinan que es regular, finalmente se tiene que 6 estudiantes que conforman el $7.5 \%$ lo encuentran como alto. Al realizar el análisis del desinterés e 
indisciplina en el aula se presenta a 32 estudiantes que conforman el $40.0 \%$ opinan que es bajo, luego se tiene a 44 estudiantes que corresponden al 55.0\% lo analizan como regular, finalmente se tiene a 4 estudiantes que corresponden al 5.0\% lo evalúan como alto. Al hacer mención a las conductas inadecuadas se pudieron demostrar a 22 estudiantes que corresponden al $27.5 \%$ lo consideran como bajo, luego se pudo demostrar que 52 estudiantes que corresponden al $65.0 \%$ lo encuentran como regular y 6 estudiantes que integran al 7.5\% lo analizan como alto. Al realizar el análisis del bullying escolar se tiene a 24 estudiantes que corresponden al $30.0 \%$ opinan que es bajo, luego se tiene a 50 estudiantes que integran el $62.5 \%$ lo ubican como regular y 6 estudiantes que representan al $7.5 \%$ lo consideran como alto.

\section{Tabla 4}

Frecuencia del aprendizaje en los estudiantes

\begin{tabular}{lcc}
\hline \multicolumn{1}{c}{ Nivel } & fi & Aprendizaje \\
\hline Deficiente & 19 & $\%$ \\
Regular & 30 & $23.8 \%$ \\
bueno & 31 & $37.5 \%$ \\
Total & 80 & $38.8 \%$ \\
\hline
\end{tabular}

Fuente: Instrumento aplicado a los estudiantes

\section{Figura 2}

\section{Barra del aprendizaje en los estudiantes}

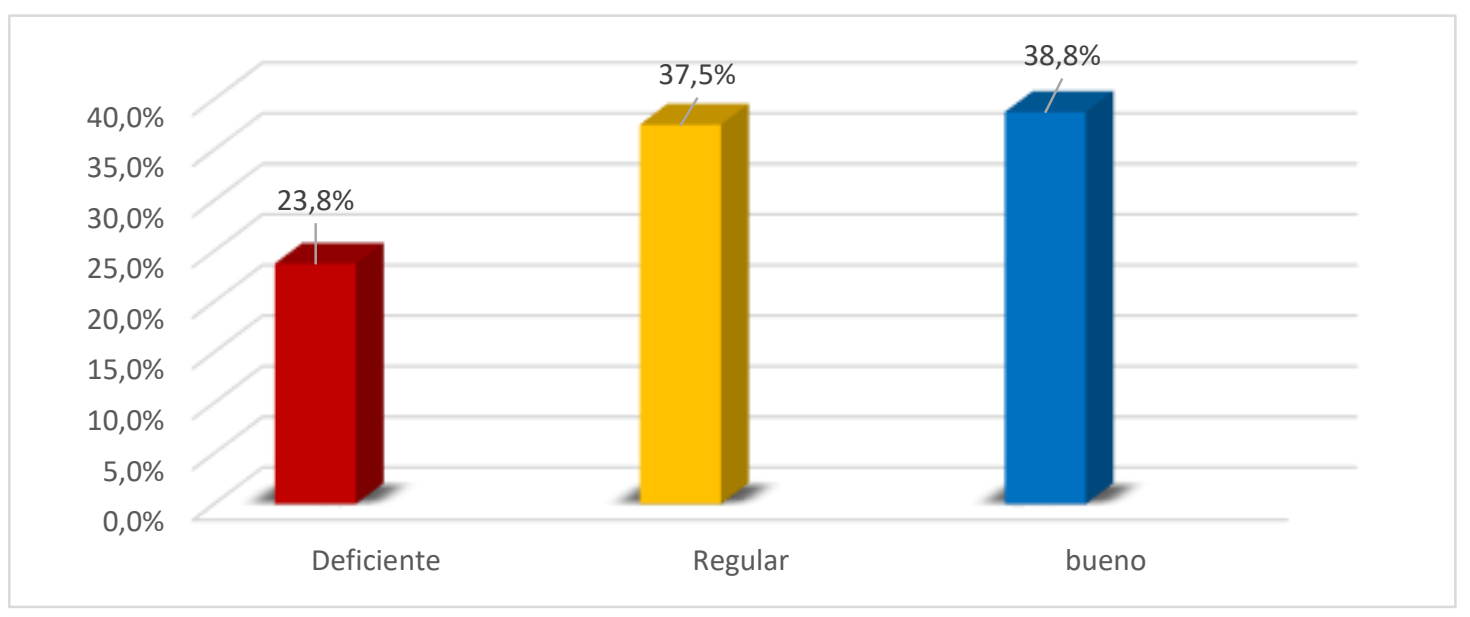

\section{Interpretación}

Por medio del análisis realizado de la aplicación del instrumento a los estudiantes se tiene para el aprendizaje a 19 estudiantes que corresponden al $23.8 \%$ se ubican en el nivel 
deficiente, luego se tiene a 30 estudiantes que corresponden al $37.5 \%$ opinan que se ubican en el nivel regular y 31 estudiantes que integran el $38.8 \%$ se ubican en el nivel bueno.

\section{Análisis inferencial y prueba de hipótesis:}

\section{Tabla 5}

\section{Normalidad de datos}

\begin{tabular}{lccc|ccc}
\hline & \multicolumn{3}{c|}{ Kolmogorov-Smirnov $^{\mathbf{a}}$} & \multicolumn{3}{c}{ Shapiro-Wilk } \\
\cline { 2 - 7 } & Estadístico & gl & Sig. & Estadístico & gl & Sig. \\
\hline Bullying escolar &, 184 & 80 &, 000 &, 889 & 80 &, 000 \\
Aprendizaje del estudiante &, 139 & 80 &, 001 &, 952 & 80 &, 004 \\
\hline
\end{tabular}

a. Corrección de significación de Lilliefors

\section{Interpretación}

Por medio del análisis realizado de la aplicación del instrumento a los estudiantes se tiene la prueba de normalidad de datos por medio del método de Kolmogorov-Smirnov ${ }^{\mathrm{a}}$, para muestras superiores a 50, encontrando un valor de significancia para el Bullying escolar de 0.000 y para el aprendizaje de los estudiantes de 0.001 , al realizar el análisis en ambos casos se puede identificar que son menores al 0.05 con lo que se demuestra que la distribución que presenta la muestra es no paramétrica y el método más adecuado para realizar el análisis de la correlación es el método de Rho de Spearman.

\section{Tabla 6}

Relación entre el bullying escolar y el aprendizaje de los estudiantes del cuarto y quinto grado de secundaria de la I.E. Micaela Bastidas - Santa Ancash año 2021

\begin{tabular}{|c|c|c|c|c|}
\hline & & & $\begin{array}{l}\text { Bullying } \\
\text { escolar }\end{array}$ & $\begin{array}{l}\text { Aprendizaje de los } \\
\text { estudiantes }\end{array}$ \\
\hline \multirow{6}{*}{ 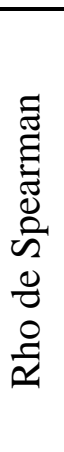 } & \multirow{3}{*}{ Bullying escolar } & $\begin{array}{l}\text { Coeficiente de } \\
\text { correlación }\end{array}$ & 1,000 &,$- 696^{* *}$ \\
\hline & & Sig. (bilateral) & . &, 000 \\
\hline & & $\mathrm{N}$ & 80 & 80 \\
\hline & \multirow{3}{*}{$\begin{array}{l}\text { Aprendizaje de los } \\
\text { estudiantes }\end{array}$} & $\begin{array}{l}\text { Coeficiente de } \\
\text { correlación }\end{array}$ &,$- 696^{* *}$ & 1,000 \\
\hline & & Sig. (bilateral) & ,000 & . \\
\hline & & $\mathrm{N}$ & 80 & 80 \\
\hline
\end{tabular}

**. La correlación es significativa en el nivel 0,01 (bilateral). 


\section{Interpretación}

Luego de realizar el análisis de los resultados obtenidos en el estudio se ha obtenido un valor de Rho de -0.696 analizado como inversa por presenta el signo negativo y considerable por el valor alcanzado, con lo que se interpreta que a menor bullying escolar se podrá tener un mejor aprendizaje de los estudiantes, con ello la dirección y docentes deben aplicar estrategias para reducir el bullying escolar y poder mejorar el desarrollo de los aprendizajes en los estudiantes.

Al realizar el análisis de la prueba de hipótesis se tiene el análisis de la significancia dando a conocer que el valor alcanzando es de 0.000 situado por debajo del 0.01 , con lo que se afirma que la relación que se presenta es inversa y significativa, procediendo al rechazo de la hipótesis nula y acetando la hipótesis planteada en el estudio que se basa en: Existe relación inversa y significativa entre el bullying escolar y el aprendizaje de los estudiantes del cuarto y quinto grado de secundaria de la I.E. Micaela Bastidas - Santa Ancash año 2021.

\section{DISCUSIÓN}

Según lo evidenciado para el objetivo general que se basa en determinar la relación entre el bullying escolar y el aprendizaje de los estudiantes del cuarto y quinto grado de secundaria de la I.E. Micaela Bastidas - Santa Ancash año 2021; luego de realizar el análisis de los resultados obtenidos en el estudio se ha obtenido un valor de Rho de -0.696 analizado como inversa por presenta el signo negativo y considerable por el valor alcanzado, con lo que se interpreta que a menor bullying escolar se podrá tener un mejor aprendizaje de los estudiantes, con ello la dirección y docentes deben aplicar estrategias para reducir el bullying escolar y poder mejorar el desarrollo de los aprendizajes en los estudiantes. Al realizar el análisis de la prueba de hipótesis se tiene el análisis de la significancia dando a conocer que el valor alcanzando es de 0.000 situado por debajo del 0.01 , con lo que se afirma que la relación que se presenta es inversa y significativa, procediendo al rechazo de la hipótesis nula y acetando la hipótesis planteada en el estudio que se basa en: Existe relación inversa y significativa entre el bullying escolar y el aprendizaje de los estudiantes del cuarto y quinto grado de secundaria de la I.E. Micaela Bastidas - Santa Ancash año 2021.

Esto coincide con los resultados de Paredes (2015), quien en su investigación sobre Bullying y rendimiento académico, en estudiantes de educación secundaria encuentra que 
no hay relación entre ambas, a un nivel significativo. En otros casos en que se estudia la relación del bullying con otras variables, se hayan resultados similares, tal es el caso de la investigación de Flores (2016) quien llegó a la conclusión de la no existencia de correlación de significancia entre las variables bullying y autoestima, también en estudiantes de educación secundaria, aun cuando encontró porcentajes mayoritarios ubicados entre los niveles muy alto y alto de bullying.

Los resultados descritos, se contraponen con los planteamientos teóricos que establecen que el bullying genera graves consecuencias como la manifestación de sentimientos de ansiedad, soledad, fobias, problemas de autoestima y, evasión al centro de estudios, rechazando el proceso de aprendizaje, esto último conllevaría al bajo nivel de aprendizaje. Para Polo del Río et ál. (2017), los efectos negativos que genera el bullying son visibles también en otras conductas como el retraimiento social y la depresión lo que afectaría su integración en el medio escolar y por tanto el normal desarrollo de los aprendizajes en los estudiantes. Para Olguín (2019) el bullying escolar contribuye al desorden y la indisciplina en el aula, esto a su vez deviene en un clima hostil que genera inestabilidad emocional tanto en estudiantes como en docentes, lo que altera los procesos formativos pues perturba al docente que no puede conducir adecuadamente el aprendizaje de sus estudiantes, por ende, estos no están en las condiciones para participar adecuadamente en la construcción de sus propios aprendizajes.

En este caso específico, el tiempo de confinamiento generada por la pandemia del COVID 19 y el desarrollo de las clases a través de medios virtuales ha anulado durante dos años académicos la interacción de los estudiantes en los espacios escolares, por lo que existe la probabilidad de que este distanciamiento en las relaciones estudiantiles haya limitado la evocación de recuerdos asociados a la práctica del bullying en las anteriores etapas presenciales de los estudios. Sería interesante estudiar la presencia del bullying en escenarios virtuales postpandemia.

Según lo evidenciado para el objetivo específico que se basa en estudiar la relación entre el comportamiento agresivo y el aprendizaje de los estudiantes del cuarto y quinto grado de secundaria de la I.E. Micaela Bastidas - Santa Ancash año 2021; luego de realizar el análisis de los resultados obtenidos en el estudio se ha obtenido un valor de Rho de -0.644 analizado como inversa por presenta el signo negativo y considerable por el valor alcanzado, con lo que se interpreta que a menor comportamiento agresivo se podrá tener 
un mejor aprendizaje de los estudiantes, con ello la dirección y docentes deben aplicar estrategias para reducir el comportamiento agresivo y poder mejorar el desarrollo de los aprendizajes en los estudiantes. Al realizar el análisis de la prueba de hipótesis se tiene el análisis de la significancia dando a conocer que el valor alcanzando es de 0.000 situado por debajo del 0.01 , con lo que se afirma que la relación que se presenta es inversa y significativa, procediendo al rechazo de la hipótesis nula y acetando la hipótesis planteada en el estudio que se basa en: Existe relación inversa y significativa entre el comportamiento agresivo y el aprendizaje de los estudiantes del cuarto y quinto grado de secundaria de la I.E. Micaela Bastidas - Santa Ancash año 2021.

Resultados similares obtiene Monelos (2015) quien estudio la violencia escolar en estudiantes de nivel secundario en La Coruña, llega a la conclusión que no existe un nivel alto de violencia estudiantil, precisando que un alto porcentaje de escolares no tienen comportamiento de agresividad; pero, un aporte importante es que detecta que un porcentaje significativo de los estudiantes guarda silencio sobre cuando es víctima del bullying. En la misma línea están los resultados del estudio realizados por Ferrel et ál (2015) pues concluyeron que no existe bullying, pero detecta dos factores de riesgo frente a los cuales hay necesidad de trabajar, la impulsividad y la soledad.

En cuanto a los hallazgos de López (2016), encuentra que la violencia escolar se pronunciaba en forma momentánea y temporal en los ambientes escolares, si bien su investigación fue de corte cuasiexperimental, la aplicación del pre test le dio alcances sobre la situación inicial del bullying antes de administrar su tratamiento para enfrentar situaciones de la violencia verbal y la discriminación, utilizando chapas, burlas, adjetivos y los comentarios.

\section{CONCLUSIÓN O CONSIDERACIONES FINALES}

Se determinó relación inversa y significativa $(\mathrm{Rho}=-0.696$; sig. $=0.000)$ entre el bullying escolar y el aprendizaje de los estudiantes del cuarto y quinto grado de secundaria de la I.E. Micaela Bastidas - Santa Ancash año 2021, dando a conocer que a menor bullying escolar se podrá tener un mejor aprendizaje de los estudiantes, de la misma se procedió con el rechazo de la hipótesis nula.

Dentro de las recomendaciones se considera a lo siguiente:

Al director de la I.E. se les recomienda realizar diagnósticos para detectar casos de bullying en los espacios escolares con la finalidad de diseñar e implementar acciones de 
intervención temprana para evitar que los estudiantes se vean afectados en su desarrollo personal y aprendizaje.

Al coordinar de tutoría promover en los docentes tutores el desarrollo de su capacidad observadora para identificar los cambios que se suscitan en los estudiantes víctimas o victimarios del bullying escolar y canalizar la intervención de los especialistas.

\section{REFERENCIAS BIBLIOGRAFICAS}

Amaya, M. C. (2017) Alternativas violentas generadas por la comunidad educativa ante el acoso escolar que afecta a los estudiantes en su diversidad. Universidad Nacional de Educación a Distancia - UNED - España. https://dialnet.unirioja.es/servlet/tesis?codigo $=129862$

Alonso, I. (2018). Propuesta de intervención para la prevención de acoso escolar en educación primaria. $\quad$ - $\quad$ UNIR. Repositorio https://reunir.unir.net/handle/123456789/6602

Baena, G. (2017). Metodología de la investigación. Serie integral por competencias (3a. ed.). Grupo Editorial Patria, S.A. de C.V. http://www.biblioteca.cij.gob.mx/Archivos/Materiales_de_consulta/Drogas_de_ Abuso/Articulos/metodologia\%20de\%20la\%20investigacion.pdf

Briones-Zambrano, F.G. (2020). Autoestima y acoso escolar en educación media pública y privada de Lima Metropolitana. Yachasun. 4(7). file://C:/Users/pc/AppData/Local/Temp/36-Article\%20Text-194-1-1020200805.pdf

Ferrel, F., Cuan, A., Londoño, Z., Ferrel, L. (2015). Factores de riesgo y protectores del bullying escolar en estudiantes con bajo rendimiento de cinco instituciones educativas de Santa Marta, Colombia. Psicogente, 18(33), 188-205. http://www.scielo.org.co/pdf/psico/v18n33/v18n33a15.pdf

Fretwell, Q. (2015). Addressing Bullying in Schools: The Perceptions, Thoughts and Beliefs of Middle-School Principals. Dissertation, Georgia State University, 2015. https://scholarworks.gsu.edu/eps_diss/132/

García, L. A. (2017, febrero 2). Muestreo probabilístico y no probabilístico. Teoría. https://www.gestiopolis.com/muestreo-probabilistico-no-probabilistico-teoria/

Hernández, R., Fernández, C. y Baptista, P. (2016). Metodología de la investigación. 6a ed. Mc Graw Hill. 
Ministerio de Educación (2017b). Orientaciones-Portal SiseVe contra la violencia escolar. https://peru.unfpa.org/sites/default/files/pubpdf/MINEDU\%20guia_orientaciones\%20siseve\%20violencia\%20escolar.pdf

Monelos, E. (2015). Análisis de situaciones de acoso escolar o bullying en centros de Educación Secundaria de La Coruña. [Tesis doctoral - Universidad La Coruña España]. https://core.ac.uk/download/pdf/61917775.pdf

Olweus, D. (1993). Bullying at school. What we know and what can do. Oxford: Blackwell.

Patterson, S. (2020). 6 Ways Educators Can Prevent Bullying in Schools. Article on the website Lesley University. https://lesley.edu/article/6-ways-educators-can-preventbullying-in-schools

Polo del Río, M. I., Mendo, S., Fajardo, F. y León del Barco, B. (2017). Una intervención en aprendizaje cooperativo sobre el perfil del observador en la dinámica bullying* Universitas Psychologica, vol. 16, núm. 1, 2017 Pontificia Universidad Javeriana, Colombia. Disponible en: http://www.redalyc.org/articulo.oa?id=64750138017

Rojas, P. (2019, 27 de setiembre). Al día, 27 niños peruanos son víctimas de violencia escolar, según último reporte del Minedu. Gestión. https://gestion.pe/peru/al-dia-27ninos-peruanos-son-victimas-de-violencia-escolar-segun-ultimo-reporte-delminedu-noticia/?ref=gesr

Sánchez, H. Reyes, C. y Mejía, K. (2018). Manual de términos en investigación científica, tecnológica y humanística. Universidad Ricardo Palma, Lima, Perú. Libro Virtual. file:///C:/Users/pc/AppData/Local/Temp/libro-manual-de-terminos-eninvestigacion.pdf 